\title{
Pathomechanisms and possible interventions in myalgic encephalomyelitis/chronic fatigue syndrome (ME/CFS)
}

\author{
Øystein Fluge, ${ }^{1,2}$ Karl J. Tronstad, ${ }^{3}$ and Olav Mella1,2 \\ 'Department of Oncology and Medical Physics, Haukeland University Hospital, Bergen, Norway. ${ }^{2}$ Department of Clinical Science and ${ }^{3}$ Department of Biomedicine, University of Bergen, Bergen, Norway.
}

M yalgic encephalomyelitis/chronic fatigue syndrome (ME/CFS) is a disease with unknown etiology, no validated specific and sensitive biomarker, and no standard approved effective treatment. ME/CFS has a profound impact on the quality of life of both patients and caregivers and entails high costs for society. The severity varies among patients who are able to participate to some extent in social life (mild), those who are mainly housebound (moderate) or bedridden (severe), and the very severely ill who are completely dependent on assistance for all daily living tasks, such as feeding or turning around in bed.

Myalgic encephalomyelitis/chronic fatigue syndrome (ME/CFS) often starts in previously healthy individuals after an infection, the most common being infectious mononucleosis (EBV). It is more frequent in women and influenced by genetic predisposition. The main symptoms are postexertional malaise (PEM), fatigue, orthostatic intolerance, cognitive disturbances, sleep problems with inadequate restitution after rest, sensory hypersensitivity with pain, and symptoms related to autonomic and immune dysfunction. The prevalence is $0.1 \%$ to $0.8 \%$, and ME/CFS must be distinguished from general fatigue, which is much more common in the population.

Historically, there has been limited scientific interest in ME/CFS. However, research efforts have increased in the last decade. Although this has led to different hypotheses, a firmly established pathomechanism is lacking.

Herein, we suggest a framework model for the initiation and maintenance of $\mathrm{ME}$ /
CFS consisting of three principal steps: (a) an initial aberrant immune response; (b) an effector system for symptom generation and maintenance; and (c) compensatory adaptations. The model and possible therapeutic opportunities are summarized in Figure 1.

\section{Immune response}

Our interest in ME/CFS started in 2007 in our cancer ward, when we observed patients with long-standing ME/CFS who got cancer and who independently reported that the cancer treatment had beneficial effects on their ME/CFS. The treatments included the cytotoxic drug cyclophosphamide and/or the monoclonal B cell-depleting anti-CD20 antibody rituximab. Our observations led to the working hypothesis that ME/CFS in a subgroup could be a variant of an autoimmune disease, often with a postinfectious onset and with a role for B cells/plasma cells and antibodies. Consequently, we decided to test relevant immunomodulatory drugs in clinical trials for ME/CFS patients.

Several findings support an autoimmune disease mechanism in ME/CFS. These include a marked female preponderance, a high frequency of autoimmunity among first-degree family members (40\%-55\% in our trials), and enriched HLA risk alleles. Among patients with an infectious onset, associations with autoimmunity-related gene variants (1) and skewed B cell receptor gene usage (2) have been demonstrated. Furthermore, elderly $\mathrm{ME} / \mathrm{CFS}$ patients have an increased risk of B cell lymphoma, especially the lowgrade marginal zone lymphomas often associated with autoimmunity or chronic infections (3). Serum B cell activating
Conflict of interest: Haukeland University Hospital has patents on the issue of B cell depletion therapy for chronic fatigue syndrome (ME/CFS), no. PCT/EP2020/074186. ØF and OM are mentioned as inventors in these applications. Copyright: (๑) 2021, American Society for Clinical Investigation.

Reference information: J Clin Invest. 2021;131(14):e150377. https://doi.org/10.1172/JCl150377. factor (BAFF) increase and gene expression studies suggesting antigen-driven $\mathrm{B}$ cell clonality (4) or altered B cell differentiation (5) may point to B cell involvement. Beneficial clinical effects have been reported after immunoadsorption to remove plasma IgG (6).

Despite promising results from our early phase II trials evaluating rituximab in $\mathrm{ME} / \mathrm{CFS}$, the subsequent multicenter, double-blind, and placebo-controlled phase III trial was negative for the outcome measures (ref. 7; additional references therein). Factors that may have influenced the outcome of the phase III trial include heterogeneity among patients, placebo mechanisms, natural symptom variation, and nonoptimal outcome measures. However, our pooled experience supports that some $\mathrm{ME} / \mathrm{CFS}$ patients do respond to rituximab and $\mathrm{B}$ cell depletion. As rituximab targets CD20-positive cells, patients whose autoantibody production occurs in CD20-positive plasmablasts would be likely responders. In the majority of patients, however, autoantibodies may be produced in CD20-negative, long-lived plasma cells not targeted by rituximab (Figure 1). These are known mechanisms in several established autoimmune diseases. However, other parts of the immune system may play important pathomechanistic roles in other subgroups of ME/CFS patients.

We also performed an open-label phase II trial with intravenous cyclophosphamide, in which half the patients reported clinical responses lasting several years (8). Cyclophosphamide has broad effects on several subsets of lymphocytes, and the mechanism for benefit in ME/CFS could involve the antiproliferative effects inhibiting B cell activation to plasmablasts (Figure 1). Although there were no severe side effects, cyclophosphamide can induce infertility, and a broader use in ME/CFS patients is problematic due to toxicity concerns. Further, we have seen symptom improvement in ME/CFS patients using 

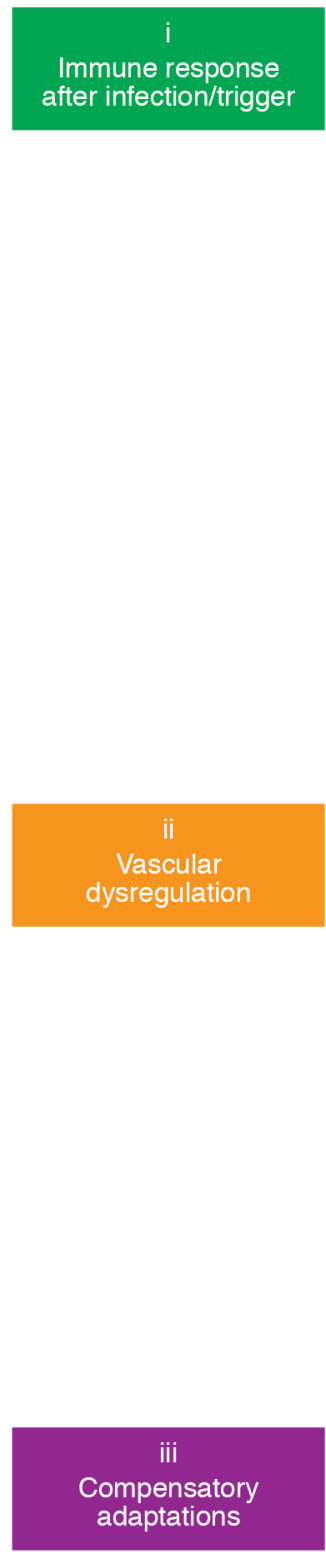
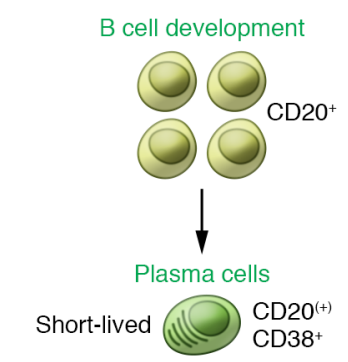

Long-lived
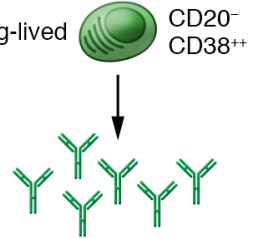

Pattern of autoantibodies after infection?

Persistent, affecting vascular function

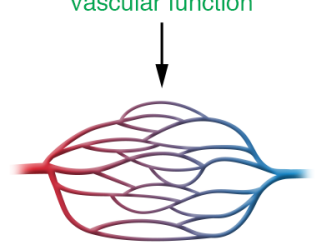

Endothelial dysfunction Large and small arteries

Arteriovenous shunting

Impaired oxygen extraction Impaired venous tone and return Preload failure

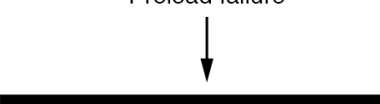

Impaired autoregulation of

blood flow on exertion

Tissue hypoxia

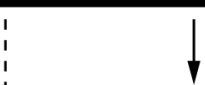

Autonomic adaptations Maintain vascular homeostasis Increased sympathetic tone

Metabolic adaptations Maintain energy balance

$$
\text { i }
$$$$
7
$$

- . . - $\rightarrow$ Symptoms

PEM

Fatigue

Exercise intolerance

POTS

Orthostatic intolerance

Cognitive

Gastrointestina
Possible targets or strategies for clinical trials

B cell depletion Anti-CD20 antibody

Cytotoxic drugs Cyclophosphamide

Plasma cell survival factors Anti-BAFF antibody

Plasma cells Anti-CD38 antibody Proteasome inhibition

Immunoglobin manipulation FcRn targeting Immunoadsorption IVIG
Acetylcholinesterase inhibition

Cognitive techniques Diets and supplements Activity pacing bortezomib, which mainly targets plasma cells, but this proteasome inhibitor is also associated with toxicity concerns.

If our interpretations regarding B cells/ plasma cells and autoantibodies in ME/CFS hold true, plasma cell targeting and immunoglobulin manipulation are therapeutic opportunities to be explored in approved clinical studies. Possible trials could include drugs that reduce serum IgG by targeting
Fc receptor neonatal (FcRn), anti-CD38 antibodies to target long-lived plasma cells or anti-BAFF antibody to target disrupted B cell homeostasis (Figure 1).

\section{Impaired autoregulation of blood flow}

How are these proposed immune disturbances and autoantibodies translated to the recognizable clinical picture of $\mathrm{ME} / \mathrm{CFS}$,
Figure 1. Proposed model for ME/CFS pathomechanisms. We suggest three principal steps underlie the initiation and maintenance of ME/ CFS. (i) Immune response after infection serves as a triggering event, with a role for $B$ cells/plasma cells and autoantibodies in the underlying pathology. (ii) The vascular system and possibly GPCRs are potential targets for autoantibodies, which may affect endothelium or neurovascular control and autonomic small nerve fibers. The autoantibodies could be pathogenic IgGs or functional autoantibodies that normally occur after infection, but persist and fail to resolve over time. This disturbed homeostasis involves endothelial dysfunction in large and small arteries, impaired venous return and preload failure, and arteriovenous shunting, presumed to result in impaired autoregulation of blood flow and tissue hypoxia on exertion. (iii) Secondary compensatory efforts may add to the clinical presentation and symptoms. They include autonomic adaptations, often with increased sympathetic tone, and metabolic adaptations aiming to restore energy supply. Possible strategies for clinical trials targeting these pathways are also indicated.

with PEM as the cardinal symptom? We believe that the clinical symptoms in $\mathrm{ME}$ / CFS suggest inadequate autoregulation of blood flow according to the demands of tissues, resulting in tissue hypoxia. This is associated with lactate accumulation from limited exertion, in some patients even at rest.

There is growing evidence for endothelial dysfunction in ME/CFS, affecting large arteries, assessed by flow-mediated dilation (FMD), and small arteries, assessed by postocclusive reactive hyperemia (refs. 9, 10, and Figure 1). FMD mainly reflects the release of nitric oxide (NO) from endothelial cells in response to shear stress in vessel walls. However, patients had intact ability to dilate adequately from endothelium-independent vasodilation when given sublingual nitroglycerin. The endothelial dysfunction was not associated with laboratory markers of endothelial dysfunction seen in cardiovascular disease, which argues for a different mechanism, possibly related to an abnormal immune response (10).

Head-up tilt testing using extracranial Doppler measurements of carotid and vertebral arteries has shown reduced cerebral blood flow (11), which may contribute to PEM and cognitive dysfunction. In a recent study assessing ME/CFS patients with upright cardiopulmonary exercise test (CPET) and invasive mon- 
itoring, a subgroup of the patients had reduced right atrial pressure and venous return (preload failure), with reduced cardiac output and reduced peak oxygen uptake on exertion (12). Other ME/CFS patients showed evidence of microcirculatory disturbances with impaired peripheral oxygen extraction, compatible with arteriovenous (AV) shunting and possibly related to neurovascular dysregulation and small fiber neuropathy (12). Impaired oxygen extraction in muscles during exercise tests has also been demonstrated by noninvasive measurements (13). According to the CPET test results, the circulatory disturbances could not be explained by deconditioning in patients with orthostatic intolerance and reduced cerebral blood flow upon head-up tilt testing (14) nor in patients with exercise intolerance and low biventricular filling pressures or impaired AV oxygen extraction (12). It would, however, be expected that physical deconditioning and other effects of long-lasting disease may contribute to symptom worsening.

Endothelial dysfunction with inadequate flow regulation to meet the demands of tissues, reduced venous tone and return, and reduced cardiac output on exertion as well as AV shunting with reduced peripheral oxygen extraction would all result in tissue hypoxia, which we believe may be a common pathomechanistic denominator in ME/CFS (Figure 1). Several two-day CPET studies have shown that ME/CFS patients have ventilatory anaerobic threshold at a lower workload compared with healthy subjects, especially on day two (15), which is a manifestation of PEM. PEM often occurs with a time lag after exertion and may be sustained for days and weeks. One may speculate that some symptom-generating factors are pathologically reinforced by exercise. Moreover, patient reports of transient symptom improvement from oxygen inhalation, from nitroglycerin-mediated vasodilation (briefly alleviating the "brain fog"), or from saline infusions (to increase volume and venous pressure) may correspond with poor vasoregulation. Evidence of neuroinflammation in ME/CFS, associated with lactate accumulation and microglia activation (16), could also be related to impaired autoregulation of blood flow and brain tissue hypoxia caused by mental or physical exertion.

\section{Compensatory adaptations}

For decades, autonomic dysfunction in ME/CFS has been repeatedly demonstrated (17). Predominantly, increased sympathetic tone and altered sympathovagal balance have been reported; see, e.g., J. Słomko et al. (18). We interpret the autonomic disturbances and increased sympathetic output as probable secondary and compensatory adaptations to inefficient blood flow regulation upon exertion. Alternatively, in some patients, there could be a primary autonomic pathomechanism with increased sympathetic tone.

Several studies have reported metabolic changes in ME/CFS patients, e.g., A. Germain et al. (19) and Ø. Fluge et al. (20). The results suggest altered utilization of substrates for energy metabolism, such as increased use of amino acids and fatty acids for tricarboxylic acid cycle (TCA) fueling, with reduced glucose and pyruvate oxidation. Impaired pyruvate dehydrogenase $(\mathrm{PDH})$ function due to increased expression of PDH kinases (PDKs) (20) may indicate chronic activation of physiological metabolic programs that normally protect cellular energy (ATP) supply under demanding conditions, such as endurance exercise, hypoxia, or starvation. Metabolic adaptations, aiming to maintain and restore energy supply, may be caused by an underlying tissue hypoxia on exertion in $\mathrm{ME} / \mathrm{CFS}$. This restriction will compromise homeostasis and promote energy strain and corresponding metabolic responses.

\section{Variant of autoimmune mechanism?}

In established autoimmune diseases, pathogenic IgGs often associate with complement activation, inflammation, and tissue injury. These features are not characteristic of ME/CFS. We suggest that a variant of an autoimmune mechanism affects the autonomic control of blood vessel tone and flow autoregulation. ME/CFS overlaps clinically with postural orthostatic tachycardia syndrome (POTS), orthostatic intolerance, fibromyalgia, and complex regional pain syndrome. We speculate that these illnesses could be associated with a pattern of autoantibodies occurring after triggers such as systemic infections (21), but not resolving properly over time. In ME/CFS, a persistent functional autoantibody pattern could disturb blood vessel autoregulation and lead to secondary metabolic and autonomic adaptations (Figure 1).

To this end, functional GPCR autoantibodies may be involved (22). Agonistic autoantibodies to $\beta 2$ adrenergic receptors ( $\beta 2 \mathrm{ARs})$ and muscarinic 3 receptors have been demonstrated in orthostatic hypotension (23). In POTS, an autoimmune basis has been suggested by the presence of several functional autoantibodies toward GPCRs affecting blood pressure and heart rate regulation, such as antibodies to $\alpha 1 \mathrm{AR}, \beta 1 \mathrm{AR}$, and $\beta 2 \mathrm{AR}$ (24), and also toward angiotensin II type 1 receptor. Autoantibodies to GPCRs, including adrenergic and muscarinic receptors, have been investigated in ME/CFS (25), and anti- $\beta 2 \mathrm{AR}$ antibodies were proposed to have an important role (26). Although GPCR autoantibodies are also present in healthy individuals, it is possible that they contribute in regulatory networks associated with different physiological states and with disease (27).

A recent study using rapid extracellular antigen profiling (REAP) to assess autoantibodies against the "exoproteome" (secreted and extracellular proteins) after COVID-19 infection showed a myriad of autoantibodies with functional impairment of many important immune-related and receptor molecules (28). Similar broad autoantibody responses may possibly occur after other systemic infections that could trigger $\mathrm{ME} /$ CFS, such as infectious mononucleosis. In a study of CFS risk after infectious mononucleosis among adolescents, $13 \%, 7 \%$, and $4 \%$ met the Fukuda diagnostic criteria for CFS after 6, 12, and 24 months, respectively (29). Such decreasing risk over time could be compatible with an initial pattern of diverse regulatory autoantibodies perturbing function, normally resolving over time, but in some patients persisting and contributing to $\mathrm{ME} / \mathrm{CFS}$.

\section{Conclusions}

Our proposed pathomechanistic model (Figure 1) is compatible with the lack of obvious histologic inflammation in tissue samples from ME/CFS, lack of overt organ damage, and the potential for recovery - sometimes spontaneous and without sequelae. Future research should focus on the natural course of ME/CFS over time to identify the mechanisms that induce and 
maintain disease, find targets for intervention, and specifically aim to elucidate immune dysregulation and patterns of autoantibodies with mechanisms for circulatory disturbances.

In our model, clinical symptoms of ME/CFS are related primarily to the inadequate autoregulation of blood flow yielding tissue hypoxia on exertion, but are also influenced by the compensatory adaptations from increased sympathetic output and from metabolic shifts. We speculate that cognitive techniques, which are reported to help subgroups of patients, might act by modulating the sympathetic output. If so, one would expect a greater benefit for patients with less ongoing immune activation and less vascular dysregulation, but with main symptom contributions from the secondary autonomic adaptations. Conversely, patients with active immune disturbance and ongoing vascular dysregulation as the main symptom generators would have less impact from cognitive intervention, although psychosocial support and coping strategies may still have a beneficial impact on their quality of life.

In conclusion, we suggest that $\mathrm{ME} /$ CFS in a subgroup of patients is a variant of an autoimmune disease, with a role for $\mathrm{B}$ cells/plasma cells and a pattern of autoantibodies emerging after infection and persisting over time. Key symptoms may result from the consequent functional disturbance in blood flow autoregulation causing tissue hypoxia on exertion and associated autonomic and metabolic responses to maintain energy homeostasis.

Finally, there is growing concern for patients with "long COVID." Research is needed to determine whether the symptoms, which may resemble those of ME/CFS, are caused by subtle organ damage from the viral infection or whether subgroups of "long haulers" actually have a postinfectious immune disturbance and pathomechanism similar to those in ME/CFS.

\section{Acknowledgments}

The research group for ME/CFS at Haukeland University Hospital receives funding from the Kavli Trust. The rituximab and cyclophosphamide trials with substudies received funding from the Research Council of Norway, the Norwegian Regional
Health Trusts, the Norwegian Ministry of Health and Care Services, the MEandYou Foundation, the Norwegian ME Association, and the legacy of Torstein Hereid.

Address correspondence to: Øystein Fluge, Department of Oncology and Medical Physics, Haukeland University Hospital, Jonas Lies vei 65, N-5021 Bergen, Norway. Phone: 47.55972010; Email: oystein. fluge@helse-bergen.no.

1. Steiner S, et al. Autoimmunity-related risk variants in PTPN22 and CTLA4 are associated with $\mathrm{ME} / \mathrm{CFS}$ with infectious onset. Front Immunol. 2020;11:578.

2. Sato W, et al. Skewing of the B cell receptor repertoire in myalgic encephalomyelitis/chronic fatigue syndrome [published online March 29, 2021.]. Brain Behav Immun. https://doi. org/10.1016/j.bbi.2021.03.023.

3. Chang $\mathrm{CM}$, et al. Chronic fatigue syndrome and subsequent risk of cancer among elderly US adults. Cancer. 2012;118(23):5929-5936.

4. Milivojevic M, et al. Plasma proteomic profiling suggests an association between antigen driven clonal B cell expansion and ME/CFS. PLoS One. 2020;15(7):e0236148.

5. Nguyen CB, et al. Whole blood gene expression in adolescent chronic fatigue syndrome: an exploratory cross-sectional study suggesting altered B cell differentiation and survival. $J$ Transl Med. 2017;15(1):102.

6. Scheibenbogen $\mathrm{C}$, et al. Immunoadsorption to remove beta2 adrenergic receptor antibodies in Chronic Fatigue Syndrome CFS/ME. PloS One. 2018;13(3):e0193672.

7. Fluge $\mathrm{O}$, et al. B-lymphocyte depletion in patients with myalgic encephalomyelitis/chronic fatigue syndrome: a randomized, double-blind, placebo-controlled trial. Ann Intern Med. 2019;170(9):585-593.

8. Rekeland IG, et al. Intravenous cyclophosphamide in myalgic encephalomyelitis/chronic fatigue syndrome. An open-label phase II study. Front Med (Lausanne). 2020;7:162.

9. Newton DJ, et al. Large and small artery endothelial dysfunction in chronic fatigue syndrome. Int J Cardiol. 2012;154(3):335-336.

10. Sørland K, et al. Reduced endothelial function in myalgic encephalomyelitis/chronic fatigue syndrome-results from open-label cyclophosphamide intervention study. Front Med (Lausanne). 2021;8:642710.

11. van Campen C, et al. Cerebral blood flow is reduced in $\mathrm{ME} / \mathrm{CFS}$ during head-up tilt testing even in the absence of hypotension or tachycardia: a quantitative, controlled study using Doppler echography. Clin Neurophysiol Pract. 2020;5:50-58.

12. Joseph $P$, et al. Insights from invasive cardiopulmonary exercise testing of patients with myalgic encephalomyelitis/chronic fatigue syndrome[published online February 10, 2021]. Chest. https://doi.org/10.1016/j.chest.2021.01.082.
13. Vermeulen RC, Vermeulen van Eck IW. Decreased oxygen extraction during cardiopulmonary exercise test in patients with chronic fatigue syndrome. J Transl Med. 2014;12:20.

14. van Campen C, et al. Deconditioning does not explain orthostatic intolerance in ME/CFS (myalgic encephalomyelitis/chronic fatigue syndrome). J Transl Med. 2021;19(1):193.

15. Stevens S, et al. Cardiopulmonary exercise test methodology for assessing exertion intolerance in myalgic encephalomyelitis/chronic fatigue syndrome. Front Pediatr. 2018;6:242.

16. Mueller C, et al. Evidence of widespread metabolite abnormalities in Myalgic encephalomyelitis/chronic fatigue syndrome: assessment with whole-brain magnetic resonance spectroscopy. Brain Imaging Behav. 2020;14(2):562-572.

17. Freeman R, Komaroff AL. Does the chronic fatigue syndrome involve the autonomic nervous system? Am J Med. 1997;102(4):357-364.

18. Słomko J, et al. Autonomic phenotypes in chronic fatigue syndrome (CFS) are associated with illness severity: a cluster analysis. J Clin Med. 2020;9(8):2531.

19. Germain A, et al. Metabolic profiling of a myalgic encephalomyelitis/chronic fatigue syndrome discovery cohort reveals disturbances in fatty acid and lipid metabolism. Mol Biosyst. 2017;13(2):371-379.

20. Fluge $\mathrm{O}$, et al. Metabolic profiling indicates impaired pyruvate dehydrogenase function in myalgic encephalopathy/chronic fatigue syndrome. JCI Insight. 2016;1(21):e89376.

21. Rivera-Correa J, Rodriguez A. Divergent roles of antiself antibodies during infection. Trends Immunol. 2018;39(7):515-522.

22. Skiba MA, Kruse AC. Autoantibodies as endogenous modulators of GPCR signaling. Trends Pharmacol Sci. 2021;42(3):135-150.

23. Li H, et al. Agonistic autoantibodies as vasodilators in orthostatic hypotension: a new mechanism. Hypertension. 2012;59(2):402-408.

24. Fedorowski A, et al. Antiadrenergic autoimmunity in postural tachycardia syndrome. Europace. 2017;19(7):1211-1219.

25. Loebel M, et al. Antibodies to $\beta$ adrenergic and muscarinic cholinergic receptors in patients with chronic fatigue syndrome. Brain Behav Immun. 2016;52:32-39.

26. Wirth K, Scheibenbogen C. A unifying hypothesis of the pathophysiology of myalgic encephalomyelitis/chronic fatigue syndrome (ME/CFS): recognitions from the finding of autoantibodies against $\beta 2$-adrenergic receptors. Autoimmun Rev. 2020;102527.

27. Cabral-Marques O, et al. GPCR-specific autoantibody signatures are associated with physiological and pathological immune homeostasis. Nat Commun. 2018;9(1):5224.

28. Wang EY, et al. Diverse functional autoantibodies in patients with COVID-19 [preprint]. https:// doi.org/10.1101/2020.12.10.20247205. Posted on medRxiv December 12, 2020.

29. Katz BZ, et al. Chronic fatigue syndrome after infectious mononucleosis in adolescents. Pediatrics. 2009;124(1):189-193. 